\title{
Exogenous Orientation of Attention to the Center of Mass in a Visual Search Task
}

\author{
Max Kailler Smith ${ }^{1} \cdot$ Marcia Grabowecky ${ }^{1}$
}

Published online: 24 December 2019

(C) The Psychonomic Society, Inc. 2019

\begin{abstract}
Anne Treisman's scientific career included broad-ranging contributions that advanced our understanding of the attentional mechanisms that people rely on to make sense of the world. In this paper, we describe results from a visual-search paradigm first developed by Grabowecky and Treisman (Grabowecky, 1992). Their design exploited known feature-search asymmetries (Treisman \& Gormican, 1988) to investigate the role of a center of mass (CoM) mechanism in determining the initial locus of visual-spatial attention in visual search. The original experiment supported the hypothesis that CoM influences initial orienting of visual-spatial attention, as targets near the CoM of a multi-element array were detected more quickly than targets distant from the CoM. These findings were replicated in a follow-up experiment using a different feature-search asymmetry, with eye-tracking added to verify central fixation. We also investigated whether CoM had any influence on pop-out search, and found no evidence that it does. Surprisingly, the effect of position of the search array on the CoM suggested that CoM may be computed independently for elements contained within each visual hemifield. Whereas our work on CoM with Treisman was initiated within an earlier theoretical context, the present results are also compatible with contemporary theoretical advances; both the early results and the new results can be integrated within current ways of thinking about attention and pre-attentive mechanisms.
\end{abstract}

Keywords Visual search · Attention

\section{Introduction}

Anne Treisman's contributions to our understanding of attention are profound. Along with her many other achievements and insights, Treisman made critical discoveries by cleverly constructing behavioral paradigms to isolate processes involved in the detection, selection, parsing, and integration of visual information. Together with other converging methods, Treisman used response-latency and errors in visual search asymmetries (e.g., Treisman \& Gormican, 1988) as a means to investigate early visual processing.

In the late 1980's and early 1990's, I (Grabowecky) was working on my dissertation with Anne Treisman and Lynn Robertson. I had collected visual search data from patients with unilateral neglect and we observed a preattentive effect of display configuration on visual search in these patients (Grabowecky, Robertson, \& Treisman, 1993).

Marcia Grabowecky

grabowecky@northwestern.edu

1 Northwestern University, Evanston, IL, USA
Patients searched a diamond-shaped array placed at the body midline, which appeared either alone, or with additional "flanking" elements that grouped with one of two types of distractor elements. We were investigating whether elements that were neglected could nonetheless help to group and segment the distractors in an array and thus make search easier. We did not observe an advantage of grouping and segmentation as we had hypothesized, but instead observed that the effect of adding flanking elements depended on their position in the array. Adding flankers to the ipsilesional ("good") side slowed search for contralesional targets (on the "bad" side). Unsurprisingly, flanking elements on the neglected (contralesional) side did not seem to help much, although across patients they did produce a small, but significant improvement in search latency. What surprised us was that the presence of bilateral flankers dramatically reduced the cost of the ipsilesional flankers. When the flankers were changed to black triangles, which grouped with the search array only by proximity because the flankers shared no features with either targets or distractors, the same pattern of results was observed in three patients with unilateral neglect (Grabowecky, 1992). 
What was going on here? Around the same time, a visual search model by a UC Berkeley computer science student was presented in a lab meeting. He had arbitrarily used the center of search arrays as the starting point for his visual search model. I speculated that the visual system might similarly use a central default starting point for attentional search. A literature search indicated that the center of mass $(\mathrm{CoM})$ or center of gravity of a peripheral array played a role in rapid deployment of saccadic eye movements, which tended to land at the center of the array (corrected for cortical magnification) (e.g., Coren \& Hoenig, 1972; Coëffé, \& O'Regan, 1987; Findlay, 1982; Ottes, Van Gisbergen, \& Eggermont, 1984). The pattern of brain lesions in the patients with unilateral neglect, but more importantly the brain structures that were spared across patients suggested that the superior colliculus was one candidate structure to mediate a CoM computation. The observations that saccadic eye movement systems were influenced by the CoM suggested that a likely neural substrate of the CoM was the frontal-collicular system. This system has also been implicated in orienting overt and covert attention. Rizzolatti and colleagues proposed an influential premotor theory of attention (Rizzolatti, Riggio, Dascola, \& Umiltá, 1987) that identified attention, and in particular attentional orienting, with the eye movement system (Rizzolatti et al., 1987; Kustov \& Robinson, 1996; but see Smith, \& Schenk, 2012 for an alternative view).

The role of the CoM in visual attention may help to explain why patients with unilateral neglect ignore half of whatever visual information they are attending (e.g., Driver \& Halligan, 1991; Ladavas, 1987; Robertson, Eglin, \& Knight, 2003). In order to neglect one half of a visual object or region of space, patients with unilateral neglect must first determine the midline of an attentional reference frame. The CoM provides a mechanism for determining the center of a visual array, and together with other stimulus attributes such as body-centered and environmental up and down determine the reference frame for attentional orienting and unilateral neglect (Lamb \& Robertson, 1988; Rock, 1956; Robertson, Palmer, \& Gomez 1987; Robertson, 1995).

Treisman and I hypothesized that: 1) a center of mass calculation might provide a default location for the direction of both attention and eye movements in patients with unilateral neglect 2) that the influence of the display configuration that we observed for the patients with unilateral neglect would also be observed in healthy young people, and would occur in the absence of eye movements, and 3) if the CoM was a default locus for attention, that targets requiring attention for their detection would be detected more quickly at the CoM than targets away from the CoM, and that this pattern should occur only when attention was needed for detection.

According to Feature Integration Theory (e.g., Treisman \& Gelade, 1980; Treisman, 1985), the presence of a feature such as the slash in a Q creates activation in an orientation feature map, and the circle $(\mathrm{O})$ activates both curvature and closure maps. Detecting a Q among O's can be accomplished by readout of the appropriate orientation map without attention to the location of the target feature (Treisman \& Souther, 1985). In this case detection should be rapid regardless of the target location in the search array. In contrast, when the target is defined by the absence of a feature, for example, a target $\mathrm{O}$ among Q's, because the target is specified by the absence of the slash in the $\mathrm{Q}$, no unique feature map activation can signal target presence, and thus attentional search would be required for its detection. In this case of a feature-absent target, targets located at the CoM should be more quickly and accurately detected than targets located away from the center of the array. Visual-spatial attention is necessary to bind the slash and the $\mathrm{O}$ at each distractor location to detect the location where an $\mathrm{O}$ is present without a slash. If the initial orienting of visual-spatial attention is influenced by the display configuration and CoM, then a target $\mathrm{O}$ appearing at the $\mathrm{CoM}$ should be detected faster than targets away from the center. Note that our manipulation of display configuration differs from the way in which configural effects are usually understood, primarily operating by enhancing or diminishing target salience by local feature contrast (e.g., Pomerantz, Sager, \& Stoever, 1977; see Treisman 1982 for a discussion of both aspects of configural effects).

We presented healthy young participants with visual arrays as illustrated in Figure 1 (Grabowecky, 1992). Participants reported the presence or absence of a target Q in O's (a popout search) or for a target $\mathrm{O}$ in Q's (an attention-demanding search) in separate blocks with the block order counterbalanced across participants. In order to equate visibility of targets while stimulus arc positions varied, targets appeared in one of 4 possible fixed locations in the display (see Figure $1 \mathrm{~A}$ ), but at different relative positions within the distractor arc that surrounded the targets (see Figure 1 B-D). Targets never appeared at the ends of the stimulus arcs. Across both conditions, participants fixated the cross at the center of the display and the stimulus presentation was brief $(150 \mathrm{~ms})$ to discourage eye movements. Our results supported the predictions from the center of mass hypothesis (see Figure 2). Central target locations were detected faster than targets that were further from the CoM and there was an interaction between feature-present/feature-absent search type and target location that was driven by the feature-absent search condition (attention demanding search). The minimum accuracy on feature-absent trials was $80 \%$ for participant's data to be included, and the average accuracy was $93 \%$. As would be expected, accuracy for feature-present-trials was higher at $97 \%$. These data provided tentative support for a role of the $\mathrm{CoM}$ in visual search in healthy young participants and for preservation of this computation in patients with unilateral neglect. A variant of the CoM hypothesis was examined by Shuren and colleagues using a line bisection task (Shuren, 


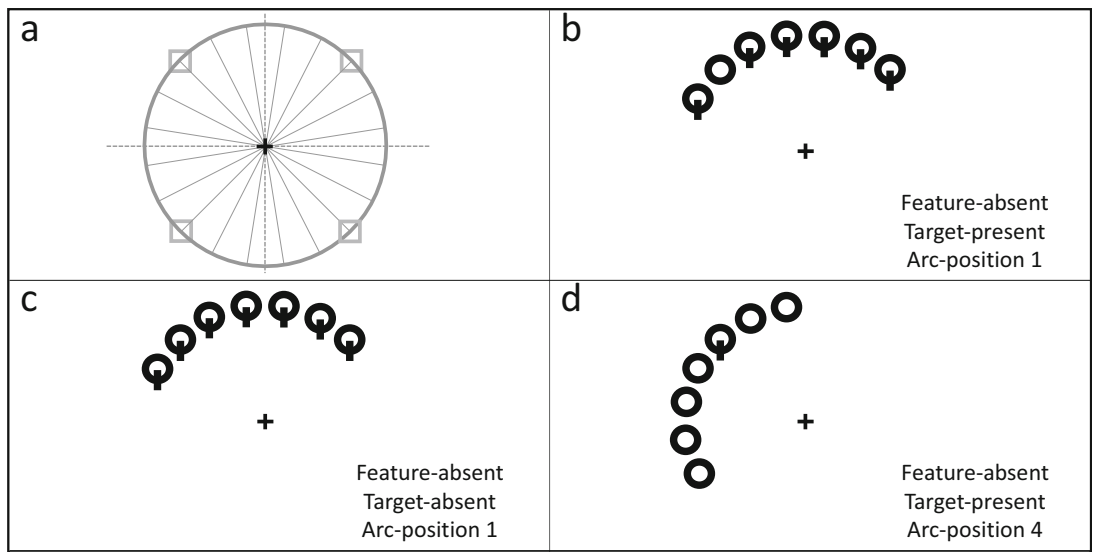

Fig. 1 Illustration of stimulus configuration. (A) The invisible circle (radius $=5^{\circ}$ visual angle) on which stimulus arcs appeared. The four grey boxes represent the four possible target locations for both experiments. Horizontal and vertical dashed lines indicate the horizontal and vertical meridians. (B-D) Illustrate the of 7-elements stimulus arcs. (B) An

Jacobs, \& Heilman, 1997). They found that healthy participants were influenced by the CoM, which was manipulated by thickening one side of a line. When participants performed a line bisection, their bisection points deviated towards the thickened portion of the line.

More recent investigations into the relationship between $\mathrm{CoM}$ and saccadic programming support the hypothesis that the CoM influences both eye movements and the initial locus of visual-spatial attention (e.g., Klein, Christie, \& Morris, 2005). A global averaging calculation across a region of the visual field spanning approximately $10^{\circ}$ appears to be automatic and determines the endpoint of short-latency (express) saccades (Aitsebaomo \& Bedell, 2000; Chou, Sommer, \& Schiller, 1999; Van der Stigchel \& Nijboer, 2011). If the CoM naturally attracts attention, then it is possible that it functions as a means to determine the center of an attentional reference frame (Lamb \& Robertson, 1988; Rock, 1956; Robertson, Palmer, \& Gomez 1987; Robertson, 1995).

Largely independently of the research we have described above, a substantial volume of research was refining our understanding of attentional mechanisms. Evidence for a fundamental distinction between exogenous and endogenous example of a feature-absent search target-present trial with a target in arc- position 1. (C) An example of a feature-absent search/target-absent trial in arc-position 1 where the target location contains a distractor. (D) An example of a feature-present search/target-present trial in arc-position 4. (reproduced from Grabowecky,1992)

attention was emerging (e.g., Posner, 1980; Posner \& Cohen, 1984, Posner \& Petersen, 1990). The developing understanding of the nature and time course of different attentional processes and the functional roles that they might play were also being investigated in the context of inhibition-ofreturn (IOR, see Wang and Klein, 2010, for a review of IOR) and attentional-capture paradigms that suggested automatic attentional orienting towards some stimulus attributes (e.g., Yantis \& Jonides, 1984; see Ruz \& Lupiáñez, 2002 and the commentaries for a review).

Work on visual search demonstrated that an exogenous spatially informative peripheral cue presented prior to a search display speeds feature detection more than a spatially uninformative cue (Briand \& Klein, 1987; Briand, 1998). Interestingly, in feature conjunction search, conjunction detection was faster as well as more accurate (i.e. occuring with fewer illusory conjunctions) when a spatially informative peripheral cue was presented compared to when a spatially uninformative peripheral cue was presented. The conjunction search cuing effect was larger than the disjunction (single feature) search cuing effect suggesting that the mechanisms supporting automatic, visual orienting to the periphery are important for veridical binding
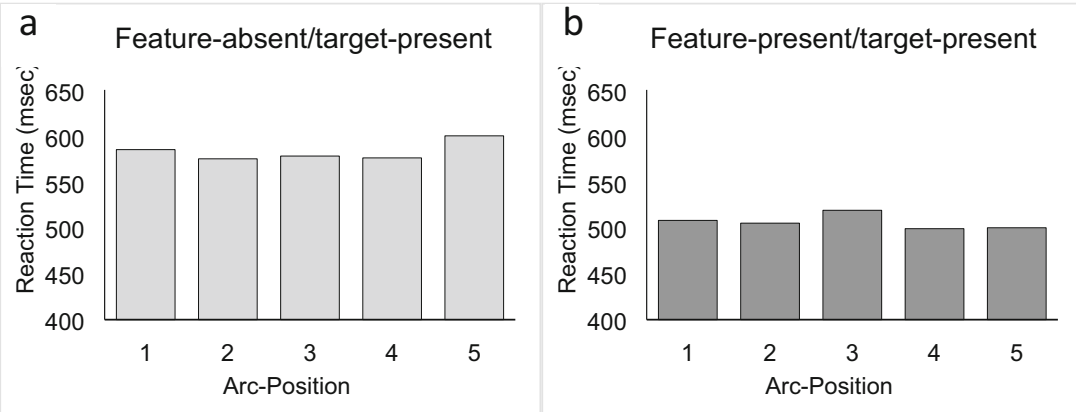

Fig. 2 Effect of arc-position on search latency. (A) Feature-absent search/target present trials, and (B) feature-present search/target present trials reaction times by arc-position, ordered from 1 to 5 with arc-position 1 indicating arcs furthest from the horizontal meridian (reproduced from Grabowecky, 1992) 
of features (Briand \& Klien, 1987; Prinzmetal, Presti, \& Posner, 1986; Treisman \& Schmidt, 1982; Treisman, 1985). RTs increase as a target appears further from the cue location (Tsal, 1983), which suggests a graded target detection benefit akin to an attentional spotlight requiring time to be moved across a visual display (e.g., Eriksen \& Hoffman, 1972). Endogenous cues presented centrally in a display did not show the same cuing difference between conjunction and disjunction search, suggesting that endogenous attentional orienting is not as important for veridical feature binding (Briand \& Klein, 1987; Prinzmetal, Presti, \& Posner, 1986; Briand, 1998).

Given the role of exogenous orienting of spatial attention in feature binding and subsequent perception, it is important to understand stimulus and task-related constraints that influence exogenous spatial orienting. We sought to replicate and extend the prior results by using a different feature-search asymmetry, verifying eye position with eye tracking, and analyzing results in additional ways. Contrasting feature-present and featureabsent search provides a method for investigating interactions between feature-based exogenous cuing and configurationbased exogenous cuing.

We hypothesize that a preattentive exogenous orientation of visual-spatial attention is cued by a CoM calculation for potential objects in a visual array, or what Wolfe and Bennett (1997, p. 40) called "shapeless collections of attributes" that are akin to the object files proposed by Kahneman \& Treisman (1984). Although the feature collections do not have a specific shape in the preattentive object file, they nonetheless have a configuration, a spatial location, and a CoM. From our perspective, none of these aspects of the object file need be specifically identified at this stage. These potential objects may consist of continuous surfaces or collections of features or objects; we focus on the latter case here. We sought to replicate the CoM effect observed by Grabowecky (1992), and to extend it to a different feature-search asymmetry, C's and O's (curvature, closure and line terminators; Treisman \& Gormican, 1988).

We used eye tracking to verify that participants maintained fixation during the brief stimulus presentation time that was used to discourage eye movements in the earlier experiments. We also wished to examine whether the CoM and feature popout might interact in orienting attention, or whether they reflect separate attentional cues. Given evidence that feature detection can occur without localization (Treisman \& Souther, 1985) and that salient features attract attention (e.g., Nothdurft, 2002), CoM and feature salience effects may be independent processes. We used a feature-search asymmetry paradigm to address these questions.

\section{Experiment}

To investigate the relationship between CoM and feature popout, we analyzed reaction time (RT) and errors for each target location for both feature-present and feature-absent search for target-present and target-absent trials. We hypothesized that the feature-absent targets at or near the CoM would be detected more quickly than targets further from the center, and that this pattern would not appear for feature-present search targets. In order to carefully examine this point, we performed contrast analyses using a quadratic polynomial to fit the data in order to determine whether the pattern of CoM benefit could be observed even to a small degree in feature-present search.

\section{Method}

\section{Participants}

Twenty-four undergraduate students (13 female, 20 righthanded) at Northwestern University gave informed consent to participate in the experiment. Given that the earlier experiments found a CoM effect with between 10 and 14 participants, we anticipated that 24 participants would ensure sufficient statistical power for this experiment. Participants were given partial course credit for their participation. All participants had normal or corrected-to-normal vision. Four participants were excluded; one for excessive sleepiness and three due to failure of eye-tracker calibration. All participants were tested in a normally lit room.

\section{Stimuli}

Participants were instructed to search for a target $\mathrm{C}$ among distractor O's or for a target $\mathrm{O}$ among distractor C's (see Figure 3). Stimuli were black on a white background to minimize screen persistence and afterimages after the display offset. Stimuli were presented in arcs that were 7 items long and appeared on an invisible circle with a radius of $5^{\circ}$ visual angle from a central fixation cross $\left(0.25^{\circ}\right.$ visual angle radius). O's and C's subtended $1^{\circ}$ visual angle. C's were defined by a $60^{\circ}$ gap. As in the original experiments, target locations were fixed at the $45^{\circ}, 135^{\circ}, 225^{\circ}$ and $315^{\circ}$ positions on the invisible circle and the location of the distractors varied around the target locations so that the target position was either in the $2^{\text {nd }}, 3^{\text {rd }}, 4^{\text {th }}, 5^{\text {th }}$, or $6^{\text {th }}$ location in the stimulus arc, with targets in the $2^{\text {nd }}$ location when the arc was farthest from the horizontal meridian (see Figure $3 \mathrm{~A}-\mathrm{B}$ ) and targets in the $6^{\text {th }}$ location when the stimulus arc was closest to the horizontal meridian (see Figure $3 \mathrm{~F}$ ). The $1^{\text {st }}$ and $7^{\text {th }}$ locations contained only distractors to ensure that all target locations had flanking elements. The gap in the $\mathrm{C}$ always pointed outward relative to the arc. Targets appeared on half of the trials. On target-absent trials, the stimulus arcs consisted of only distractors and each trial was matched to the configuration of a target-present trial. There were 40 trials in each block (4 quadrants x 5 relative 


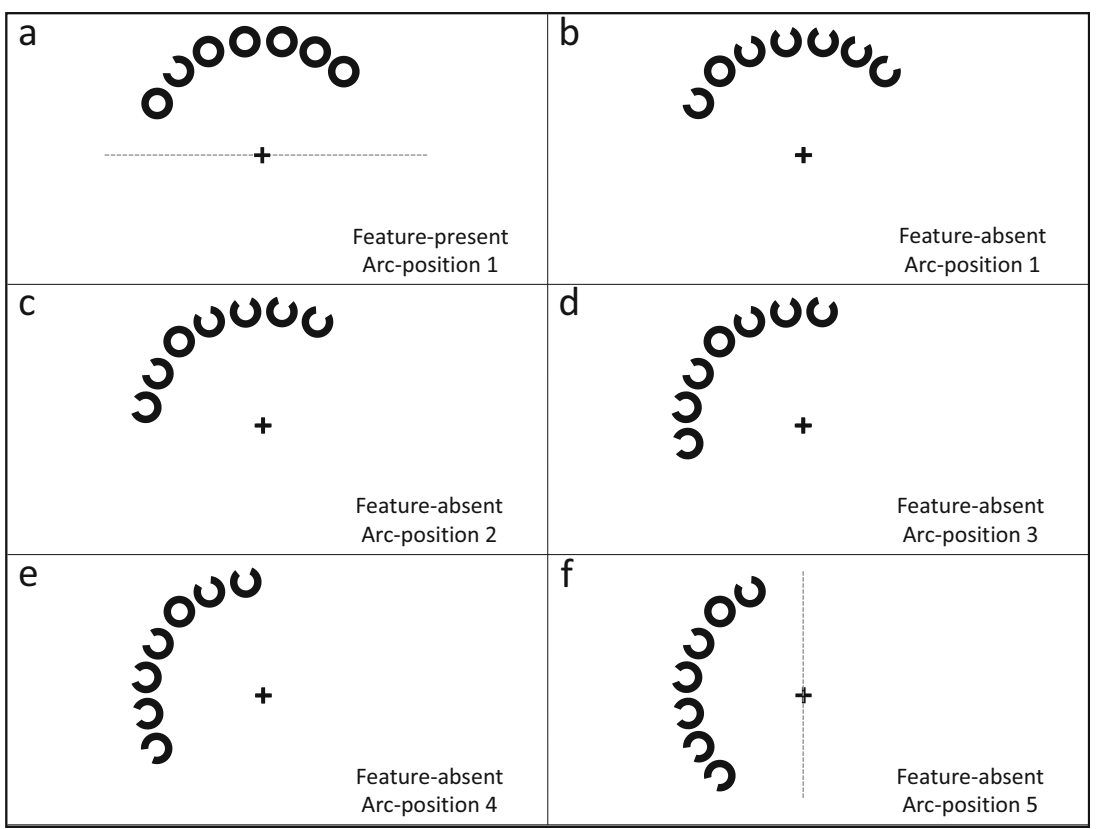

Fig. 3 Panels depict the 5 possible arc-positions using upper left quadrant target-present trials for illustration. (A) An example of a feature-present/ target-present trial with arc-position 1 . The dotted line illustrates the horizontal meridian and was not present in the display. (B-F) Examples of

target locations x target-present/target-absent). Participants received either the feature-present search trials followed by the feature-absent search trials or vice versa in counterbalanced order. Counterbalancing was not affected by participant exclusions.

Stimuli were presented on a 21.3" LCD Monitor with a resolution of 1600 by 1200 pixels and a refresh rate of 60 Hz. The experiment was controlled by a Lenovo G500 computer (Windows 8.1 operating system, $1600 \mathrm{mHz}$ ). The experiment was scripted in Matlab (2013a) using the Psychtoolbox 3.0.14 beta (Brainard, 1997; Kleiner, Brainard, \& Pelli, 2007). Participants sat at a viewing distance of $83.5 \mathrm{~cm}$ from the computer screen and maintained stable head position with a chin and forehead rest. Left eye position was monitored with an EyeLink 1000 system (SR Research Ltd., Mississauga, Ontario, Canada) set to a sampling rate of $1000 \mathrm{~Hz}$ (accuracy: 0.5; precision: 0.01 RMS). Manual responses were collected with left or right arrow key presses on a standard computer keyboard.

\section{Procedure}

Participants were verbally instructed at the beginning of the experimental session to press the left arrow key with their right index finger as quickly as possible if the target was present, and to press the right arrow key with their right middle finger if the target was absent. Participants were told to fixate the central cross for the duration of each trial. Prior to calibration of the eye tracker, participants rested their heads on the chin feature-absent/target-present trials in arc-positions $1,2,3,4$, and 5 . Feature-present/target-absent and feature-absent/target absent trials replicated these displays with distractors in the target positions.

and forehead rest and were asked to keep their heads in the same position for the duration of the experiment. Each experimental session began with two practice blocks of 20 trials followed by 16 blocks of 40 experimental trials ( 8 featurepresent blocks and 8 feature-absent blocks). The second practice block was always the same feature-present/absent condition as the first experimental block, and the first practice block corresponded to the other condition.

At the beginning of each block, participants viewed an instruction screen that reminded them of the current search target. Trials began with a $500 \mathrm{~ms}$ fixation cross. The search stimulus arc then appeared for $150 \mathrm{~ms}$, followed immediately by a blank screen. Participants were asked to respond as quickly as possible without making too many errors. When participants made an error they heard a $500 \mathrm{~ms} 500 \mathrm{~Hz}$ tone via headphones as feedback. The next trial began $1000 \mathrm{~ms}$ after the participant's response. Participants were given an opportunity to take a short break every 20 trials to minimize fatigue.

\section{Analysis}

Maintenance of fixation for the duration of each trial was determined using SR Research DataViewer saccade/fixation detection algorithm with default parameters (SR Research Ltd., Mississauga, Ontario, Canada). Within-trial fixations detected outside of a central circular area with a $1^{\circ}$ visual angle radius were classified as fixation loss trials and those trials were excluded from analysis. Responses 3.5 times the median absolute deviation (in the unit of standard deviation estimated 
with the normal assumption) from the median RT were excluded from analysis ( Ley, Klein, Bernard, \& Licata, 2013; Rosenmani, 2013).

\section{Results}

Accuracy and RTs (for correct trials) for target-present and target-absent trials were analyzed separately. Accuracy was calculated by computing the proportion correct then converting proportion correct to the corresponding probit transform.

A two-way repeated-measures analysis of variance (ANOVA) was conducted on target-present trials with RT as the dependent variable with search type (feature-present vs. feature-absent) and target position (ordered from 1 to 5 with the number indicating the position of the arc in the display; see Figure 3) as factors. There was a main effect of RT on search type with faster RT's for feature-present target search $(\mathrm{M}=$ 504.53, $\mathrm{SD}=106.08$, se $=9.22$ ) compared to feature-absent target search $(\mathrm{M}=549.90, \mathrm{SD}=114.27$, se $=9.22) ; \mathrm{F}(1,19)=$ $12.08, \mathrm{p}=0.0025$ (See Figure $4 \mathrm{~A}-\mathrm{B})$. There was also a main effect of target position, $F(1,19)=10.72, p=0.003$ (See Figure 4). Additionally, there was an interaction effect between search type and target position, $\mathrm{F}(1,19)=4.65, \mathrm{p}=$ 0.044 .

A similar two-way repeated measures ANOVA was conducted on target-absent trials. There was a main effect of RT on search type with faster feature-present search $(\mathrm{M}=519.09$, $\mathrm{SD}=111.07$, se $=8.05$ ) compared to feature-absent target
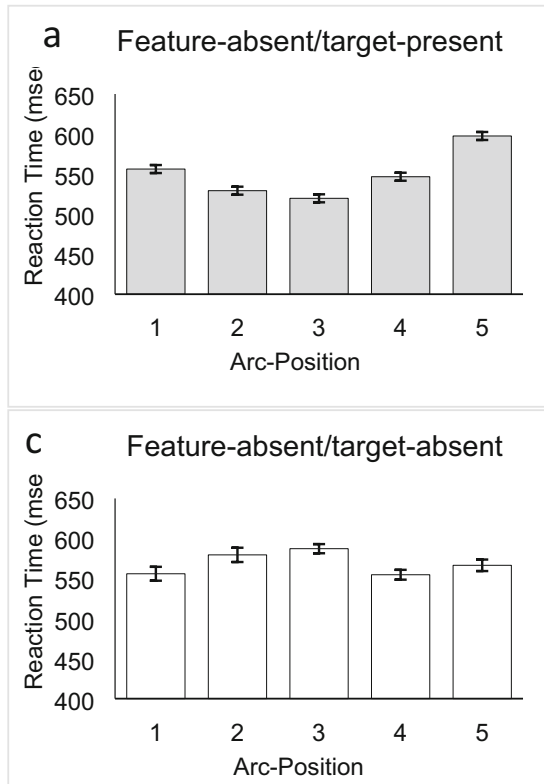

Fig. 4 Effect of arc-relative target location on search latency. (A \& C) feature-absent search. (B \& D) feature-present search. Target-absent arcpositions indicate distractor-only equivalents of each arc-position. search $(\mathrm{M}=559.34, \mathrm{SD}=113.68$, se = 8.05); $\mathrm{F}(1,19)=$ $7.52, p=0.012$ (See Figure 4 C-D). There was no main effect of arc-position, $\mathrm{F}(1,19)=3.37, \mathrm{p}=0.81$. There was no interaction effect between search type and arc-position, $\mathrm{F}(1,19)=$ 2.03, $\mathrm{p}=0.17$.

Accuracy was higher for feature-present target trials $(\mathrm{M}=$ $0.92, \mathrm{SD}=0.12, s e=0.02$ ) than for feature-absent target trials $(\mathrm{M}=.86, \mathrm{SD}=.13, \mathrm{se}=.025), \mathrm{F}(1,19)=6.69, \mathrm{p}=0.028$, significantly dependent on target position, $\mathrm{F}(1,19)=6.08, \mathrm{p}=$ 0.023 , with a significant interaction effect between search type and target position, $F(1,19)=7.90, p=0.011$. The pattern of these results shows no evidence of a speed-accuracy tradeoff (Figure 5).

The significant search type by target position interaction for the target-present trials suggests the presence of a CoM effect on feature-absent trials, but not on feature-present trials.

Specifically, the feature-absent trials show a U-shaped pattern (Figure $4 \mathrm{~A}$ ) reflecting a possible graded detection benefit for the target occupying the centroid that is not there for the feature-present trials, which show a flat pattern (Figure 4 B). To statistically test the presence of a Ushaped pattern, we applied quadratic polynomial contrast analyses to the RT data from both feature-absent and feature-present conditions. As expected, the quadratic trend was significant for the feature-absent condition, $\mathrm{F}(1,19)=24.12, \mathrm{p}<.00001$, partial $-\eta^{2}=0.559$, but nonsignificant for the feature-present condition $\mathrm{F}(1,19)=$ $1.48, p=0.23$, partial- $\eta^{2}=0.072$. These results are consistent with the interpretation that in the absence of feature pop-out, targets closer to the CoM are detected faster.
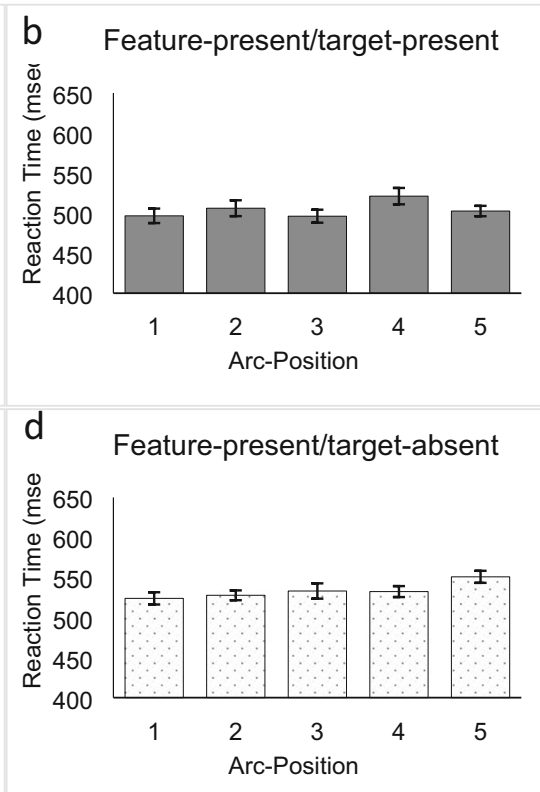

Position 3 indicates target-present trials when the target is in the center of the display arc. Error bars indicate standard error of the mean for within-subjects design (see Morey, 2008). 
a Feature-absent/target-present

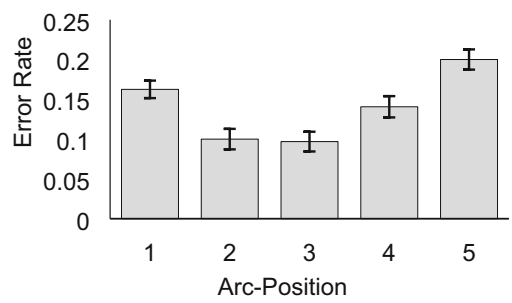

C Feature-absent/target-absent

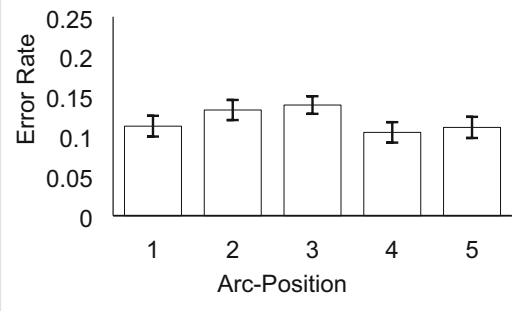

Fig. 5 Effect of arc-relative target location on search error rate. (A \& C) feature-absent search. (B \& D) feature-present search. Target-absent arcpositions indicate distractor-only equivalents of each arc-relative target

Accuracy showed a significant inverted U-shaped pattern for the feature-absent target-present trials, $F(1,19)=37.88, p$ $<0.00001$, partial- $\eta^{2}=0.665$, and no significant quadratic trend for the feature-present trials, $\mathrm{F}(1,19)=1.89, \mathrm{p}=0.18$, partial- $\eta^{2}=0.091$, again showing no evidence of a speed accuracy trade-off. The RT pattern observed for the feature present-search may be consistent with lateral masking which increases as target positions are further from the ends of the arc. Although these positions did not significantly differ from one another, search was slower in the center. Outer target positions were not on the ends of the arc, but it is possible that these positions still benefitted from having fewer neighboring elements on one side. If this type of masking did occur for the feature-absent search as well, then the benefit of the central positions in target search must have been strong enough to offset it.

Because the stimulus arcs varied around the fixed target positions (see Figure 3), some arcs were presented entirely or almost entirely within one hemifield, whereas others straddled the vertical meridian. Grouping tends to be stronger within hemifield (e.g., Choo \& Franconeri, 2010; Clevenger \& Beck, 2014), and object-related receptive fields in inferotemporal cortex are large but restricted to a single hemisphere (see Sweeny, Grabowecky, Paller, \& Suzuki, 2009 for an example in face perception). Thus, it is possible that the computation of CoM may weigh within-hemifield information more heavily. If so, then feature-absent target detection should be faster for the arcs that straddle the vertical meridian and cross hemifields because the targets were near the CoM of the elements within the same hemifield, and also potentially b Feature-present/target-present

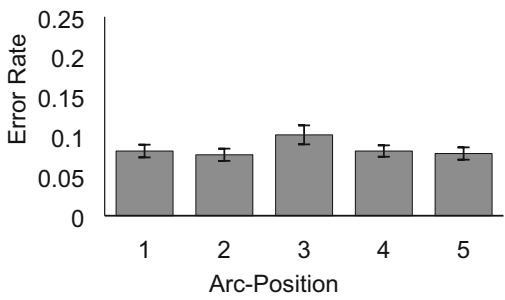

d Feature-present/target-absent

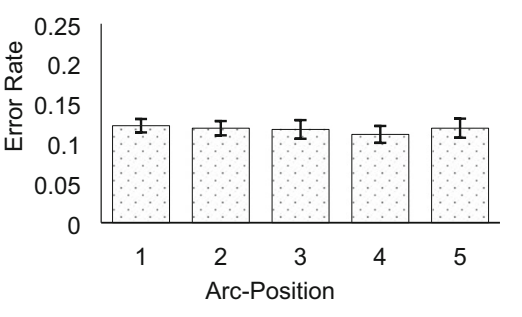

position. Position 3 indicates the center of the display arc. Error bars indicate standard error of the mean for within-subjects design (see Morey, 2008)

reducing the array size. Specifically, we compared two arcs that were completely within the same hemifield (see Figure 3 E, F) with the corresponding arcs that crossed the vertical meridian and were in two hemifields (see Figure $3 \mathrm{C}, \mathrm{B}$ ). As expected, RTs were faster for the cross-hemifield arcs $(\mathrm{M}=$ 542.76, $\mathrm{SD}=113.75, s e=5.02)$, than for the within-hemifield $\operatorname{arcs}(\mathrm{M}=572.12, \mathrm{SD}=126.93, \mathrm{se}=8.07), \mathrm{t}=3.10, \mathrm{p}=$ 0.0058 . Accuracy was also higher for cross-hemifield arcs $(\mathrm{M}=.87, \mathrm{SD}=.14, \mathrm{se}=.01)$ than for within hemifield arcs $(\mathrm{M}=.83, \mathrm{SD}=.13, \mathrm{se}=.007), \mathrm{t}=-3.228, \mathrm{p}=0.0044$. These results suggest an effectively larger array size and/or a greater crowding or masking effect for the within hemifield arcs compared to the cross-hemifield arcs.

\section{Discussion}

\section{Summary}

This experiment replicates the 1992 results that the center of mass selectively influences detection of attention-demanding feature-absent search targets. The current experiment used a different feature asymmetry, finding a closed circle (with an absence of terminators in circles with a gap (an $\mathrm{O}$ in $\mathrm{C}^{\prime} \mathrm{s}$ ) whereas the earlier experiments used the absence of a line (search for an O in Q's). In addition, we confirmed that this effect occurred in the absence of eye movements. The data suggest that the CoM is available quickly and automatically as a potential default locus for the orientation of visual-spatial 
attention, and that the CoM computation may occur within, rather than across, visual hemifields.

CoM and feature salience The CoM effect is observed when no salient feature dominates the visual array. The CoM effect may be a result of spatial averaging in a salience map (e.g., Trappenberg, Dorris, Munoz, \& Klein, 2001; Van der Stigchel \& Nijboer, 2011; see Chong and Treisman, 2003 for other forms of statistical averaging). As a result, the location of the CoM of an object configuration would create a salience peak and afford rapid detection and increased accuracy at that location. In visual arrays where a single salient feature pops out, activity associated with that feature may produce a detection benefit that supersedes or is processed independently of, and more quickly than the CoM calculation. Though pop-out leads to speedy detection, it may occur in the absence of information about spatial location (Treisman \& Souther, 1985). This is supported by similar detection accuracy and latency across all target positions for the feature-present target condition. It remains unclear how different orienting cues are prioritized, although the first available cue consistent with task demands seems likely to be the one selected. This would explain why the CoM has little effect on the rapid feature-present target search. This is consistent with the possibility that due to the blocking of feature-present target search and featureabsent target search trials, participants may have assumed a specific attentional control strategy for the feature-present search that blocked any effect of the CoM.

Saccadic averaging, CoM and Inhibition of Return Klein and colleagues (Klein, Christie, \& Morris, 2005) suggested that the population coding in oculomotor pathways that is responsible for saccadic averaging (e.g., a CoM effect) may also explain why inhibition of return (IOR) is strongest for the CoM. They demonstrated that IOR was strongest for the center of gravity when multiple distractors were present in a task that required a manual target-detection response while participants maintained central fixation. Christie and colleagues (Christie, Hilchey, \& Klein, 2013) used the same task except that participants were told to saccade to a target location immediately when the target appeared. Prior to target presentation single or multiple distractor cues appeared on the screen. Again, IOR was greatest for the CoM. These results support the idea that the CoM effect and the IOR effect may be determined by the same underlying mechanism. Pop-out may bypass this mechanism, although attention that is captured to a location by a salient irrelevant singleton creates IOR for a subsequent target at that location (Theeuwes, \& Godijn, 2002).

Visual search and exogenous and endogenous attention Exogenous and endogenous attention are dissociable, although they functionally overlap (e.g., Briand, 1998; Briand
\& Klein, 1987; Posner \& Petersen, 1990). Combining Posner cuing with feature conjunction/disjunction search demonstrates that the detection of a conjunction target is faster and that fewer illusory conjunctions are reported when a valid exogenous spatial cue appears at the target location compared to invalid exogenous spatial cues (Briand \& Klein, 1987; Prinzmetal, Presti, \& Posner, 1986; Treisman \& Schmidt, 1982). On the other hand, endogenous cuing did not produce a spatial cuing difference between disjunction and conjunction search (Briand, 1998; Briand \& Klein, 1987; Prinzmetal, Presti, \& Posner, 1986). Briand (1998) describes the exogenous system as being automatic and insensitive to expectancies, whereas the endogenous system is intentional and voluntary and is sensitive to cues with predictive validity. The two attentional systems also have different processing consequences. Both exogenous and endogenous attention change our percepts, but they do so in different ways (see Carrasco and Barbot, 2019 for a review). In particular, exogenous attention alters perceived contrast, and this enhancement can cascade to higher-level visual objects such as faces (e.g., Störmer, \& Alvarez, 2016).

CoM as a function of exogenous attention When Grabowecky and colleagues reported their results from unilateral neglect patients, they referred to the preattentive processing that was hypothesized to underlie the attentional orienting required to bind feature and location in feature-absent search (Grabowecky, Robertson, \& Treisman, 1993). They did not distinguish whether the process involved exogenous or endogenous attentional orienting, or some interaction of the two. Both the patients with unilateral neglect and our healthy young participants were volitionally searching for targets. In the case of the patients, an initial exogenous orienting to the CoM was followed by a slower endogenously driven search. For the healthy young participants, the brief display duration and small display size prevented any slower search component from being enacted. Given that the CoM is a function of stimulus configuration and produces an automatic (though not mandatory) orienting of attention, it appears to be an exogenous orienting cue (e.g., Klein, Kingstone, \& Pontefract,1992).

Is orienting to the CoM an optimal visual search strategy? Nijemnik and Geisler (2005) investigated whether humans employ ideal eye movement strategies during visual search by pitting human performance against ideal Bayesian observers with different search functions. All ideal observers were programmed to have a foveated visual system that approximated the human system via a visibility map that weighted each potential location such that locations closer to the center of the "retina" are more visible and visibility decreases as a function of eccentricity. The ideal observers used crosscorrelation template matching in which the retinal image was 
multiplied by the target template to achieve a posterior probability map of the likely locations of the target. The goal of the ideal observer was to maximize target detection accuracy in a cluttered environment.

When the ideal observer was programmed to make random initial fixations, compute posterior probability, and integrate information across fixations, the ideal observer underperformed human searchers. When the ideal observer was programmed to include IOR, the ideal observer still did not perform as well as humans. This suggested that random initial fixations were a suboptimal search strategy. When the ideal observer only fixated the maximum posterior probability, the ideal observer did about as well as human observers. However, without initial fixations to the CoM, the average distribution of fixation locations across the display differed greatly from human observers. Thus, it seems that human observers use the CoM to initiate search and IOR to bias it.

\section{Conclusion}

The CoM is a default location for shifts of exogenous visualspatial attention. Over recent decades, research has established a functional link between mechanisms that guide covert attention and contribute to saccadic programming. The current study verifies the reliability of previous research that provided evidence that the CoM calculation is an early visual process that guides covert attention in addition to guiding saccades. These data suggest that the CoM mechanism provides a fundamental way in which the visual system shifts attention to regions of space containing likely objects of interest. The CoM calculation may serve an important role in establishing attentional reference frames. A suspected mechanism underlying the CoM effects is thought to be weighted averaging which is likely to be carried out in the superior colliculus (Klein, Christie, \& Morris, 2005) which was a candidate neural substrate based on our data from patients with unilateral neglect (Grabowecky, Robertson, \& Treisman, 1993).

Our results suggest that feature pop-out, at least in the absence of overt visual-spatial orienting, appears to be uninfluenced by the CoM orienting mechanisms. At some point the cues to orientation of spatial attention, including feature salience (potentially weighted by factors such as anisotropic cortical magnification) and configural effects such as the CoM, as well as expectancy and IOR must be incorporated into an executable plan for orienting attention and the eyes. It seems likely that the cues resulting in the most rapid deployment of attention and eye movements would win. It is less clear how these exogenous attentional mechanisms would resolve conflict when competing exogenous cues are present.

Wolfe and Utochkin (2019) suggest some criteria for identifying preattentive features, some of which may guide visual selective attention. They point out that stimulus-based guidance of visual selective attention partly depends on local feature contrast. In the case of our stimuli, feature-present targets, Q's or C's, have an asymmetric feature-level contrast with the $\mathrm{O}$ distractors. In other words, the slash in the $\mathrm{Q}$ and the gap in the $\mathrm{C}$ contrast with the closed $\mathrm{O}$, but the $\mathrm{O}$ has no unique features and thus reduced feature contrast. At the same time, the stimulus arc, a potential object, contrasts with the background (i.e., the potential object has a figure-ground contrast). When the target is defined by feature-absence, an $\mathrm{O}$ in Q's or C's, only the latter contrast is available to guide attention. In this case the salience peak (e.g., Trappenberg, Dorris, Munoz, \& Klein, 2001; Van der Stigchel \& Nijboer, 2011) may identify the CoM, effectively identifying an invisible "salient" element that guides visual selective attention. In this way, the CoM may be a distinct preattentive feature, one of which we are unaware.

Open Practices Statement None of the data or materials for the experiments reported here is available, and none of the experiments was preregistered.

\section{References}

Aitsebaomo, A. P., \& Bedell, H. E. (2000). Saccadic and psychophysical discrimination of double targets. Optometry and Vision Science, 77(6), 321-330.

Brainard, D. H. (1997) The Psychophysics Toolbox, Spatial Vision, 10, 433-436.

Briand, K. A. (1998). Feature Integration and Spatial Attention: More Evidence of a Dissociation Between Endogenous and Exogenous Orienting. Journal of Experimental Psychology: Human Perception and Performance, 24(4), 1243-1256.

Briand, K. A., \& Klein, R. M. (1987). Is Posner's "Beam" the Same as Treisman's "Glue"? On the Relation Between Visual Orienting and Feature Integration Theory. Journal of Experimental Psychology: Human Perception and Performance, 13(2), 228-241.

Carrasco, M., \& Barbot, A. (2019). Spatial attention alters visual appearance. Current Opinion in Psychology, 29, 56-64.

Chong, S. C., \& Treisman, A. (2003). Representation of statistical properties. Vision Research, 43, 393-404.

Choo, H., \& Franconeri, S. (2010). Hemifield modulation of approximate number judgments. Journal of Vision, 10(7), 1207-1207.

Chou, I.-H., Sommer, M. A., \& Schiller, P. H. (1999). Express averaging saccades in monkeys. Vision Research, 39(25), 4200-4216.

Christie, J., Hilchey, M. D., \& Klein, R. M. (2013). Inhibition of return is at the midpoint of simultaneous cues. Attention, Perception \& Psychophysics, 75(8).

Clevenger, J., \& Beck, D. M. (2014). Refining the resource model: Cortical competition could explain hemifield independence. Visual Cognition, 22(8), 1022-1026.

Coëffé, C., \& O'Regan, J. K. (1987). Reducing the influence of non-target stimuli on saccade accuracy: Predictability and latency effects. Vision Research, 27(2), 227-240.

Coren, S., \& Hoenig, P. (1972). Effect of Non-Target Stimuli upon Length of Voluntary Saccades. Perceptual and Motor Skills, 34(2), 499-508. 
Driver, J., \& Halligan, P. W. (1991). Can visual neglect operate in objectcentred co-ordinates? An affirmative single-case study. Cognitive Neuropsychology, 8(6), 475-496.

Eriksen, C. W. \& Hoffman, J. E. (1972). Temporal and spatial characteristics of selective encoding from visual displays. Perception \& Psychophysics, 12, 201-204.

Findlay, J. M. (1982). Global visual processing for saccadic eye movements. Vision Research, 22(8), 1033-1045.

Grabowecky, M. (1992). Preattentive Processes Guide Visual Attention: Evidence from Normals and Patients with Unilateral Visual Neglect. University of California, Berkeley, California, 9330564.

Grabowecky, M., Robertson, L. C., \& Treisman, A. (1993). Preattentive processes guide visual search: Evidence from patients with unilateral visual neglect. Journal of Cognitive Neuroscience, 5(3), 288-302.

Kahneman, D. \& Treisman, A. (1984). Changing views of attention and automaticity. In R. Parasuraman, \& D. R. Davies (Eds.) Varieties of attention, (pp. 29-61). New York, NY: Academic Press.

Klein, R. M., Christie, J., \& Morris, E. P. (2005). Vector averaging of inhibition of return. Psychonomic Bulletin \& Review, 12(2), 295300 .

Klein, R., Kingstone, A., \& Pontefract, A. (1992). Orienting of visual attention. In K. Rayner (Ed.) Eye movements and visual cognition, (pp. 46-65). Springer, New York, NY.

Kleiner, M, Brainard, D, \& Pelli D, (2007), "What's new in Psychtoolbox-3?" Perception 36 ECVP Abstract Supplement.

Kustov, A. A., \& Robinson, D. L. (1996). Shared neural control of attentional shifts and eye movements. Nature, 384(6604), 74-77.

Ladavas, E. (1987). Is the hemispatial deficit produced by right parietal lobe damage associated with retinal or gravitational coordinates? Brain, 110, 167-180.

Lamb, M. R., \& Robertson, L. C., (1988) The processing of hierarchical stimuli: effects of retinal locus, locational uncertainty, and stimulus identity. Perception \& Psychophysics, 44: 172-181.

Ley, C., Klein, O., Bernard, P., \& Licata, L. (2013). Detecting outliers: Do not use standard deviation around the mean, use absolute deviation around the median. Journal of Experimental Social Psychology, 49, 764-766.

Nijemnik, J., \& Geisler, W. S. (2005). Optimal eye movement strategies in visual search. Nature, 7031, 387-391.

Nothdurft, H. C. (2002). Attention shifts to salient targets. Vision Research, 42(10), 1287-1306.

Ottes, F. P., Van Gisbergen, J. A., \& Eggermont, J. J. (1984). Metrics of saccade responses to visual double stimuli: two different modes. Vision Research, 24(10), 1169-1179.

Pomerantz, J. R., Sager, L. C., \& Stoever, R. J. (1977). Perception of wholes and of their component parts: some configural superiority effects. Journal of Experimental Psychology: Human Perception and Performance, 3(3), 422-435.

Posner, M. I. (1980). Orienting of attention. Quarterly Journal of Experimental Psychology, 32(1), 3-25.

Posner, M. I., \& Cohen, Y. (1984). Components of visual orienting. Attention and Performance X: Control of Language Processes, 32, 531-556.

Posner, M. I., \& Petersen, S. E. (1990). The attention system of the human brain. Annual Review of Neuroscience, 13(1), 25-42.

Prinzmetal, W., Presti, D. E., \& Posner, M. I. (1986). Does Attention Affect Visual Feature Integration? Journal of Experimental Psychology: Human Perception and Performance, 12(3), 361-369.

Rizzolatti, G., Riggio, L., Dascola, I., \& Umiltá, C. (1987). Reorienting attention across the horizontal and vertical meridians: evidence in favor of a premotor theory of attention. Neuropsychologia, 25(1), $31-40$.
Robertson, L. C. (1995). Covert orienting biases in scene-based reference frames: orientation priming and visual field differences. Journal of Experimental Psychology. Human Perception and Performance, 21: 707-718.

Robertson, L. C., Palmer, S. E., \& Gomez, L. M. (1987). Reference frames in mental rotation. Journal of Experimental Psychology Learning, Memory, and Cognition, 13: 368-79.

Rosenmani, P. (2013). Using the median absolute deviation to find outliers. Eureka Statistics.

Ruz, M., \& Lupiáñez, J. (2002). A review of attentional capture: On its automaticity and sensitivity to endogenous control. Psicológica, 23(2), 283-309.

Smith, D. T., \& Schenk, T. (2012). The premotor theory of attention: time to move on? Neuropsychologia, 50(6), 1104-1114.

Störmer, V. S., \& Alvarez, G. A. (2016). Attention alters perceived attractiveness. Psychological Science, 27(4), 563-571.

Sweeny, T. D., Grabowecky, M., Paller, K. A., \& Suzuki, S. (2009). Within-hemifield perceptual averaging of facial expressions predicted by neural averaging. Journal of Vision. 9(3), 1-11.

Theeuwes, J., \& Godijn, R. (2002). Irrelevant singletons capture attention: Evidence from inhibition of return. Perception \& Psychophysics, 64(5), 764-770.

Trappenberg, T. P., Dorris, M. C., Munoz, D. P., \& Klein, R. M. (2001). A model of saccade initiation based on the competitive integration of exogenous and endogenous signals in the superior colliculus. Journal of Cognitive Neuroscience, 13(2), 256-271.

Treisman, A. (1982). Perceptual grouping and attention in visual search for features and for objects. Journal of experimental psychology. Human perception and performance, 8(2), 194-214.

Treisman, Anne. (1985). Preattentive processing in vision. Computer Vision, Graphics and Image Processing, 31(2), 156-177.

Treisman, A. M., \& Gelade, G. (1980). A feature-integration theory of attention. Cognitive Psychology, 12(1), 97-136.

Treisman, A., \& Gormican, S. (1988). Feature analysis in early vision: evidence from search asymmetries. Psychological Review, 95(1), $15-48$.

Treisman, A., \& Schmidt, H. (1982). Illusory conjunctions in the perception of objects. Cognitive Psychology, 14(1), 107-141.

Treisman, A., \& Souther, J. (1985). Search asymmetry: A diagnostic for preattentive processing of separable features. Journal of Experimental Psychology: General, 114(3), 285-310.

Tsal, Y. (1983). Movement of attention across the visual field. Journal of Experimental Psychology: Human Perception and Performance, 9(4), 523-530.

Van der Stigchel, S., \& Nijboer, T. C. (2011). The global effect: what determines where the eyes land? Journal of Eye Movement Research, 4(2) 1-13.

Wang, Z., \& Klein, R. M. (2010). Searching for inhibition of return in visual search: A review. Vision Research, 50(2), 220-228.

Wolfe, J. M., \& Bennett, S. C. (1997). Preattentive object files: Shapeless bundles of basic features. Vision Research, 37(1), 25-43.

Wolfe, J. M., \& Utochkin, I. S. (2019). What is a preattentive feature? Current Opinion in Psychology, 29, 19-25.

Yantis, S., \& Jonides, J. (1984). Abrupt visual onsets and selective attention: evidence from visual search. Journal of Experimental Psychology: Human Perception and Performance, 10(5), 601-621.

Publisher's note Springer Nature remains neutral with regard to jurisdictional claims in published maps and institutional affiliations. 